\title{
Norois
}

Environnement, aménagement, société

223 | 2012

Villes petites et moyennes

\section{Élevage caprin, rituel hindou et réglementation sanitaire française : tradition, concertation et régulation sur l'île de La Réunion}

Goat rearing, Hindu ritual and French health rules: tradition, consultation and

regulation on the island of Reunion

Hélène Paillat-Jarousseau, Edouard Noujarede et Alain Mouchès

\section{OpenEdition}

Journals

Édition électronique

URL : https://journals.openedition.org/norois/4214

DOI : 10.4000/norois.4214

ISBN : 978-2-7535-2043-1

ISSN : $1760-8546$

Éditeur

Presses universitaires de Rennes

Édition imprimée

Date de publication : 30 mars 2012

Pagination : 93-104

ISBN : 978-2-7535-2041-7

ISSN : 0029-182X

Référence électronique

Hélène Paillat-Jarousseau, Edouard Noujarede et Alain Mouchès, «Élevage caprin, rituel hindou et réglementation sanitaire française : tradition, concertation et régulation sur l'île de La Réunion », Norois [En ligne], 223 | 2012, mis en ligne le 28 février 2014, consulté le 14 janvier 2022. URL : http:// journals.openedition.org/norois/4214; DOI : https://doi.org/10.4000/norois.4214 


\title{
Élevage caprin, rituel hindou et réglementation sanitaire française : tradition, concertation et régulation sur l'île de la Réunion
}

\author{
Goat Rearing, Hindu Ritual and French Health Rules: \\ Tradition, Consultation and Regulation on the Island of Reunion
}

\author{
Hélène Paillat-Jarousseau ${ }^{a *}$, Édouard Noujarède ${ }^{\mathrm{b}}$, Alain Mouchès ${ }^{\mathrm{c}}$
}

\footnotetext{
*Auteur correspondant

a UMR 6590 ESO-Angers CNRS Université Catholique de l'Ouest, 3 place André Leroy, BP 10808 - 49008

ANGERS cedex 01, France (hjarousseau@uco.fr)

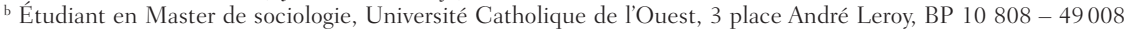
ANGERS cedex 01,France (edouard.noujarede@gmail.com)

' Laboratoire de Psychologie des Pays de la Loire - LUNAM, Université Catholique de l'Ouest, 3 place André-Leroy,

BP10808-49008 ANGERs cedex 01, France (amouches@uco.fr)
}

Résumé : Les pratiques d'élevage caprin et du rituel sacrificiel hindou de boucs, à La Réunion, sont remis en cause par l'application des règlements sanitaires de santé publique. Cet article montre que les conditions de reproduction d'un rite passent nécessairement aujourd'hui par l'acculturation et la réinvention culturelle pour produire de nouvelles normes culturelles et sociétales. Au-delà, il témoigne, à travers les autorités sanitaires et associations impliquées, de l'enjeu territorial que représente la confrontation entre une pratique religieuse et le développement d'une filière agricole.

\begin{abstract}
Based on elements of knowledge of goat rearing practices and Hindu sacrificial billy goats as well as being called into question by applying public health sanitary regulations, this article demonstrates that the conditions of reproducing a ritual need to include cultural integration and cultural reinvention to produce a definition of new social and cultural norms. Above all it demonstrates, through health authorities and the association involved, the local territorial issue is that the confrontation between religious practice and the development of an agricultural sector.
\end{abstract}

Mots clé : risque sanitaire - élevage caprin - rite religieux hindou - milieu insulaire (La Réunion) - anthropologie - développement territorial

Keywords: sanitary risk - goat rearing - Hindu ritual - insular environment (Reunion Island) - anthropology - territorial development 


\section{INTRODUCTION}

Au dernier recensement de 1999, La Réunion, département français d'Outre-mer depuis 1946, située dans l'Océan Indien, comptait 706180 habitants répartis sur une superficie de 250724 hectares. En janvier 2011, elle a été estimée à 808250 habitants (source INSEE). Société multiculturelle, elle est composée de populations issues d'Afrique, d'Inde, de Chine, de Madagascar, des Comores et d'Europe, arrivées dans l'île au cours des différentes phases de la colonisation et de développement de l'île amorcée dans la deuxième moitié du XVII ${ }^{\mathrm{e}}$ siècle. Le résultat du peuplement de l'île est une population fortement métissée, rendant impossible l'évaluation du poids réel de chacune de ses composantes (Vaxelaire, 2009) et où le plus souvent, « l'individu réunionnais incarne la pluralité ethnique et culturelle» (Live, 1999, p. 190). Par ailleurs, les Réunionnais d'origine indienne qui pratiquent l'hindouisme d'origine populaire en Inde, sont également en majorité catholiques. Ancré dans le passé colonial français, le catholicisme représente la religion dominante à la Réunion.

Le développement de l'hindouisme est directement issu de l'histoire de l'immigration indienne du temps de l'engagisme au XIX ${ }^{\mathrm{e}}$ siècle et des conditions d'établissement dans l'île de cette population venue travailler sur les grands domaines de canne à sucre. Aujourd'hui, le paysage réunionnais témoigne encore de cette histoire par la présence de temples sur le pourtour de l'île, zone des anciennes grandes plantations, que l'on nomme la ceinture cannière (figure 1 - planche I).

Le rituel sacrificiel de boucs à La Réunion est le fait de ces Réunionnais d'origine indienne et de religion hindoue. La rupture avec le pays d'origine, ainsi que l'absence de brahmanes lors de cette vague migratoire, a permis de conserver le sacrifice d'animaux dans ses formes traditionnelles (Ghasarian, 1991). Au départ, les conditions économiques des engagés ne permettaient pas l'organisation de nombreux sacrifices. Progressivement, avec l'augmentation du niveau de vie, la possibilité d'élever des animaux, de les entretenir, et de les offrir en sacrifice s'est accrue.

En France, la loi du 8 juillet 1965 sur la modernisation du marché des viandes, organise les circuits de commercialisation autour d'un réseau d'abattoirs et insiste sur la normalisation de la qualité des viandes. Dans son prolongement, la loi du 28 décembre 1966 sur l'élevage, rend obligatoire l'identification des animaux $^{1}$. Les règles sanitaires concernant la production caprine s'inscrivent dans cette logique réglementaire mise en œuvre à partir des années 1960 (Piet, 2005). À partir de cette période, des directions de Services Vétérinaires publics (DSV) sont instituées, pour répondre aux problèmes de mortalité humaine liés aux maladies et à l'alimentation des animaux. La gestion administrative du risque sanitaire se situe donc à la rencontre de la protection des consommateurs et de l'organisation du marché des viandes. Aujourd'hui, c'est principalement l'Union européenne, qui soumet une réglementation en la matière aux différents pays membres, l'État français la soumettant à son tour aux directions départementales des Services Vétérinaires (DDSV) chargées de les faire appliquer. Ceci témoigne entre autres, de la globalisation du risque sanitaire lié aux productions d'origine animale dont la question transcende aujourd'hui le cadre de l'État-nation.

Dans ce contexte, ont été définies les modalités visant à rendre plus transparent le déroulement des transactions entre les éleveurs, les consommateurs et l'administration sanitaire et fiscale ${ }^{2}$. Parmi ces modalités, la notion de traçabilité des produits animaux désigne «l'aptitude à rapporter un produit aux différentes étapes de son élaboration, afin d'en retrouver l'origine et le cheminement » (Piet, 2005). Cette définition sous-entend l'existence d'une gestion technique du risque sanitaire selon laquelle les produits d'origine animale, destinés à la consommation humaine, doivent faire l'objet de plusieurs contrôles des Services Vétérinaires à l'abattoir et à différentes étapes du processus de transformation, de manière à vérifier leur conformité aux normes de qualité sanitaire contenues dans le principe législatif de précaution.

Aujourd'hui, en l'Ile de La Réunion, la production de boucs (cabri en créole) destinée au sacrifice rituel du culte hindouiste représente la majeure partie de la production. Sachant que la production caprine est un des derniers secteurs de la production agricole locale à ne pas être administrée par les services de l'État

1. JO du 29 décembre 1966, p. 11619, Loi n 66-1005 du 28 décembre 1966 sur l'élevage.

2. Kessous E., 2006. La traçabilité généralisée : un nouveau paradigme de la consommation instrumentée par les objets communicants? communication au colloque AFS - RTf 11, Bordeaux, 5-8 septembre 2006. 
ayant en charge d'assurer la gestion du risque sanitaire, les acteurs locaux, publics et associatifs, l'appréhendent comme une réalité à transformer visant notamment à lutter contre les maladies d'origine animale transmissibles à l'homme. Parmi ces maladies transmissibles à l'homme par le caprin, la brucellose, du nom de la bactérie brucella qui en est responsable, est la plus répandue et la plus connue (Wyatt, 2009).

Selon C. Ghasarian (2002), l'étude d'une société comme la société réunionnaise, issue du passé colonial de la France, nécessite la prise en compte de trois forces interdépendantes : (i) l'acculturation par un modèle dominant qui s'impose successivement aux autres modèles culturels, (ii) la créolisation par le mélange des modèles qui aboutit à une nouvelle forme plus ou moins syncrétique, (iii) la réinvention culturelle au sens d'une reformulation résultant d'adaptations pour produire de nouvelles significations sociales et culturelles. Ces trois forces historiques se combinent et agissent également de façon permanente au sein de la société actuelle. Ainsi, partant de la présence de deux univers normatifs, celui du rituel et celui d'une agriculture organisée, l'objectif de cet article est de montrer que, dans nos sociétés contemporaines aux territoires administrés comme La Réunion aujourd'hui, l'acculturation et la réinvention culturelle demeurent les conditions nécessaires à la reproduction d'un rite qui maintienne l'identité culturelle. Pour traiter cette question, nous commencerons par étudier le système composé des actes rituels au sens de M. Mauss (1985) en cernant la place qu'y occupe l'animal. Puis, par la confrontation des normes religieuses et sanitaires, nous étudierons les possibilités d'intégration de la production sous la forme de filière en identifiant les enjeux qui s'y dessinent pour chacun des acteurs impliqués : État, éleveurs et pratiquants.

Dans la mesure où l'animal et le traitement qui lui est réservé pour sa mise à mort, sont au centre des préoccupations à propos de la norme, une partie du travail a consisté à participer à une cérémonie publique (culte au temple) (figure 2) à Saint-Louis (figure 1) et trois cérémonies privées (culte à la chapelle familiale) (figure 3) à Saint-Pierre et SainteSuzanne (figure 1) mettant en scène le sacrifice du bouc. Ce travail de type ethnographique a associé l'observation du rite et des échanges informels avec les participants et officiants. À cela, se sont ajoutés des entretiens de type sociologique avec des

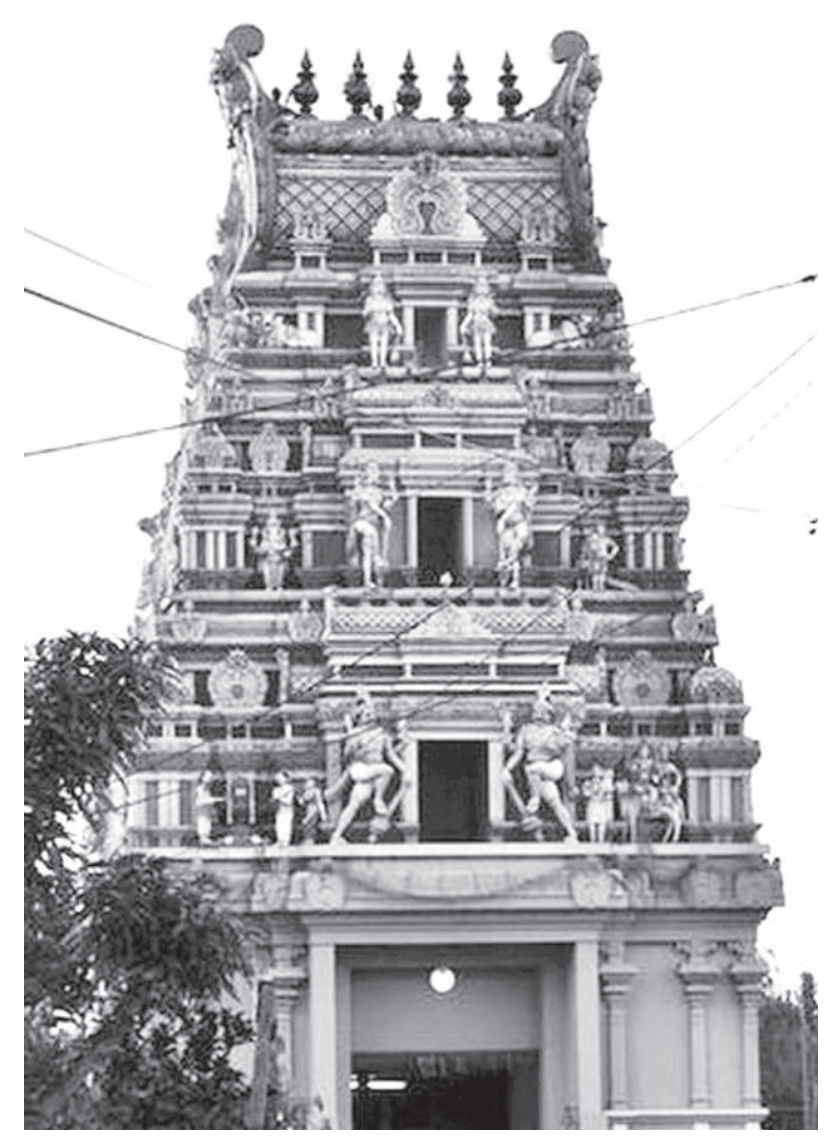

Figure 2: Photo temple public Public place of worship

prêtres et des fidèles; ceci afin de donner la lecture anthropologique du rite hindou impliqué dans le sacrifice du bouc cérémoniel et d'origine populaire à La Réunion. Des entretiens ont ensuite été réalisés avec un technicien de la coopérative de caprins de la Réunion (CPCR), ainsi qu'avec six éleveurs destinant leur production, ou partie de celle-ci, à l'utilisation religieuse, pour caractériser le « marché du rituel ». Enfin, des entretiens ont eu lieu avec des représentants locaux de l'action publique (Préfecture, direction des Services Vétérinaires, Établissement Départemental de l'Élevage), associative (Fédération Tamoul ${ }^{3}$ ), dans le but de situer la production agricole de caprins au regard du

3. La Fédération des associations et groupements religieux hindous et culturels tamouls de La Réunion est une association loi 1901, qui a été créée en 1971, visant à revaloriser la culture et la religion hindoue dans l'île; Tamoul désigne le Tamil Nadu, une région de l'Inde d'où sont originaires une grande partie des descendants d'engagés indiens, appelés malbars à la Réunion; Tamoul désigne aussi la religion et à travers elle, la préservation des rites et de la religion d'origine. 


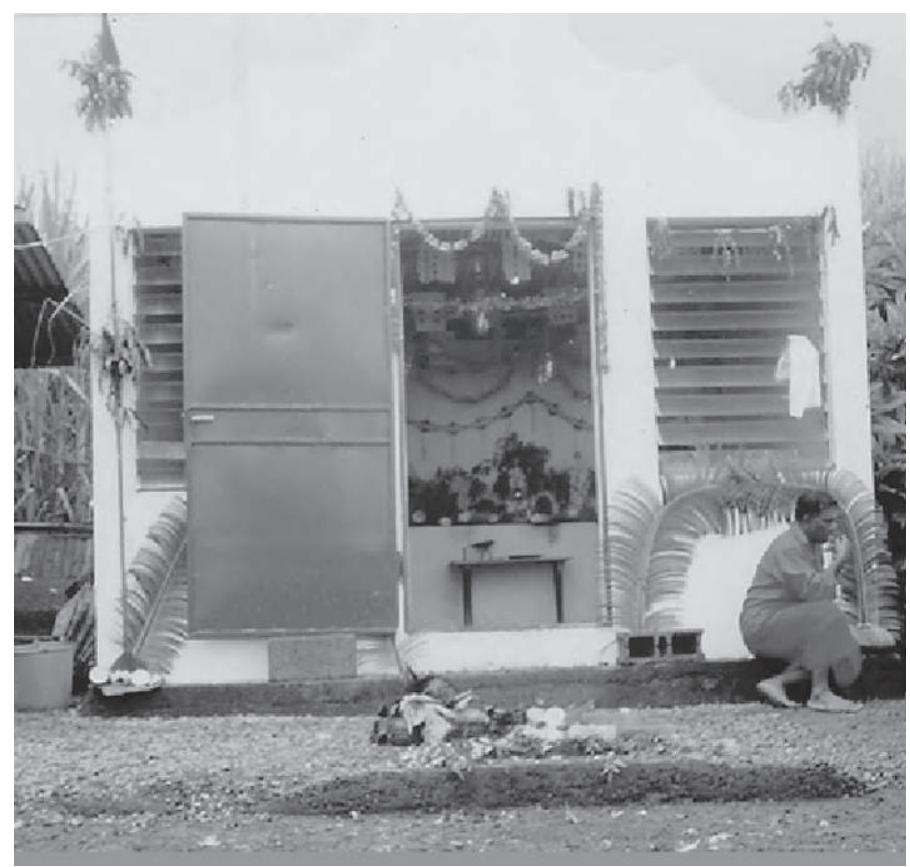

Figure 3 : Chapèl, offrandes et Kabarlom

Family place of worship (chapel in Creole), the gifts and the Kabarlom

modèle d'application des règlements sanitaires de santé publique et des enjeux religieux. Quatre personnes ont été contactées et rencontrées à plusieurs reprises parmi lesquelles le Président de la Fédération Tamoul et le Secrétaire Général de la Préfecture dont le rôle est, entre autres, d'assister l'autorité préfectorale dans la mise en ouvre des politiques nationales et communautaires de l'État.

\section{LIEUX DE CULTE ET LOGIQUE SACRIFICIELLE}

Les pratiques religieuses mettant en scène des sacrifices d'animaux se sont maintenues au cours de l'histoire à La Réunion dans un contexte socioculturel pourtant profondément acculturé. Avec l'évolution des modes de vie, les offrandes animales se sont accrues et prennent place aujourd'hui dans des cérémonies publiques et privées sur l'ensemble du territoire réunionnais. Temples (figure 2) et chapèls (figure 3) sont les lieux associés au culte. La répartition spatiale de ces lieux est présentée sur la figure 1. Dans la mesure où les manifestations concrètes du sacrifice se heurtent actuellement à la mise en œuvre des règlements sanitaires, l'ethnographie du rituel permet de comprendre et de réfléchir aux modalités d'ajustements réciproques des deux modèles culturels.

\section{Bouc cérémoniel : objet de culte privé et public}

Historiquement, à l'initiative de certains grands propriétaires de domaines de canne à sucre, la pratique hindoue du sacrifice de boucs cérémoniels par les travailleurs en provenance de l'Inde a été autorisée et facilitée en attribuant à ces derniers des parcelles de terre. Avec le démantèlement des grandes propriétés, ces temples acquièrent progressivement un caractère public; ouverts à tous les pratiquants, ils sont aujourd'hui gérés par des associations de temples (associations loi 1901) et forment avec ceux des propriétés privées familiales dits semi-publics, ce que l'on nomme communément à La Réunion, les temples à société (figure 2).

Parallèlement à ce développement, les temples domestiques appelés Chapèls (figure 3) prennent place généralement dans la cour accolée à l'habitat, et leurs dimensions sont généralement corrélées à celle de la propriété. Leur accès est privé, réservé aux membres de la famille et à son environnement relationnel proche, que peut constituer le voisinage ou des connaissances amicales.

Les formes de célébration hindoue à La Réunion peuvent donc faire l'objet d'une typologie «public/ privé » selon le lieu où elles se déroulent. La diversité des formes de cérémonies peut également s'appréhender au regard du type d'oblation qu'elles comprennent. Le culte populaire hindou distingue en effet les divinités acceptant les offrandes animales et les divinités n'acceptant strictement que les offrandes végétariennes. Cette distinction n'implique cependant aucune forme de hiérarchisation entre les divinités pour les pratiquants (Ghasarian, 1991) et se traduit en créole par les expressions i Coup / i Coup pa, Coupé faisant référence au moment du sacrifice où la tête de la victime est séparée de son corps à l'aide d'un sabre.

Généralement, les familles disposant d'un temple domestique organisent, au moins une fois par an, une cérémonie comprenant des sacrifices d'animaux appelée sèrvis ${ }^{4}$. Dans ce cas, c'est le père qui officie ou bien un prêtre si la famille le demande. Ce type

4. Sèrvis est le mot créole qui signifie cérémonie religieuse. 
de cérémonies peut cependant avoir lieu au sein de certains temples publics que l'association qui les gère se donne pour vocation d'accueillir et d'organiser à la demande des familles qui ne possèdent pas de Chapèl.

\section{Bouc cérémoniel et logique sacrificielle}

Lors des cérémonies, le bouc est d'abord signé après avoir été purifié à l'aide d'eau safranée et béni par l'encens; un marlé, collier composé d'œillets d'Inde, est placé autour de son cou. Cette étape marque la sacralisation de l'animal (figure 4). On observe alors attentivement le comportement de l'animal dans l'attente d'un signe. Lorsque l'animal se secoue, on considère que l'on peut le sacrifier. Le bouc est alors présenté au sacrificateur. Ses cornes sont tenues par un premier participant, alors que deux autres le tiennent par ses pattes arrière de manière à ce que le cou de l'animal soit présenté correctement c'est-à-dire tendu. La tête de l'animal est tranchée d'un seul geste du haut vers le bas à l'aide d'un sabre; elle est immédiatement déposée sur une feuille de bananier à côté du Kabarlom. Le Kabarlom est une petite fosse carrée creusée dans la terre, à l'intérieur de laquelle le sang de l'animal doit couler. En effet, dans la religion hindoue, le culte védique accorde à la nature un caractère divin (Ghasarian, 1991). Dans cette logique, la petite fosse représente la déesse Terre et constitue le passage obligé pour que l'offrande puisse être consommée par l'entité divine, le sang constituant un des termes de la communication avec la divinité. Ce type de cultes attribués aux divinités est envisagé comme un moyen de se prémunir contre des événements défavorables pouvant intervenir dans le quotidien profane. C'est ainsi, entre des catégories de pensée qui conçoivent l'univers de manière antagoniste selon des principes de protection et de reproduction du bon ordre des choses, que se légitiment les manifestations concrètes du culte ancestral que représente le sacrifice du bouc (figure 4).

Le sacrifice du bouc constitue donc une vision du monde et de l'univers où la relation avec le divin définit les conditions de la vie quotidienne, vis-à-vis de forces négatives source de désordre et de perturbations, mais aussi celles de la vie future en termes de réussite, de chance et de protection (Ghasarian, 1991). Le culte est envisagé par les croyants qui

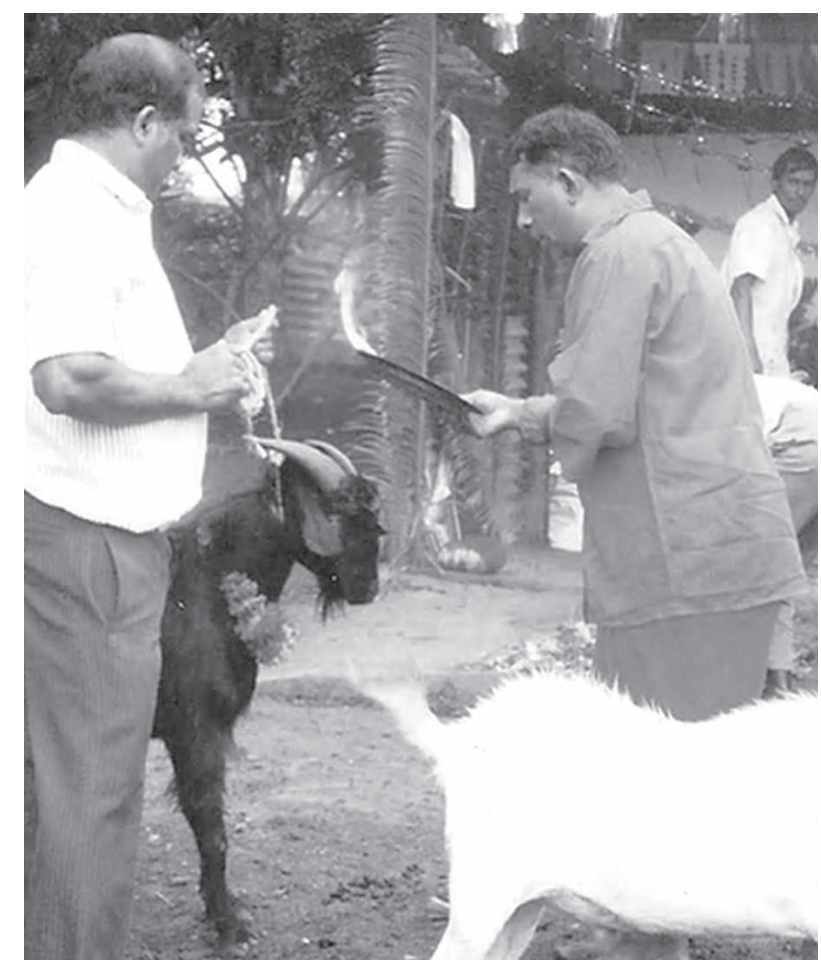

Figure 4 : Sacralisation du bouc Sacralization of billy goat

pratiquent ces sacrifices comme un moyen de se prémunir contre des événements défavorables pouvant intervenir dans leur vie comme en témoignent les propos de ce président d'association de temple rencontré "nous ici, quand on adore Karly c'est pour détruire les démons [...], nous adorons cette divinité pour qu'elle nous aide sur terre à protéger notre pays ». Selon cette logique, les ancêtres sont des esprits intermédiaires entre le monde du divin et celui de la réalité quotidienne et représentent des protecteurs. L'animal est alors envisagé comme un substitut de la personne. On coupe et on offre la tête parce que dans les représentations c'est à cet endroit que l'âme se trouve. Or c'est l'âme de l'animal qui est destinée à la divinité. Par ailleurs, la religion hindoue implique une croyance en la réincarnation. Selon ses préceptes, l'être humain a sept vies et si lors des six premières, il peut être réincarné, lors de sa septième vie, il peut l'être en «quelque chose que les gens vont chérir, offrir à Dieu justement » comme l'explique un prêtre rencontré. Ainsi, l'animal que l'on va destiner au sacrifice peut être un ancêtre, par ailleurs exemplaire. 
L'animal se retrouve ainsi au centre des rituels. Pour ce qui concerne le type d'animal, personne n'a été en mesure d'expliquer les raisons du choix du bouc au cours des enquêtes. La seule réponse a été la suivante : «nous, on se base sur les valeurs de nos grands-parents ». En d'autres termes, avec la transmission au fil des générations, les pratiques instituées gagnent en opacité, le bouc étant devenu un élément de la structure globale de l'ordre institutionnel religieux parce qu' « on a toujours fait comme ça ». Cela fait partie des éléments de la structure de communication avec le divin. Autrement dit «tout se passe donc comme si la société humaine ne pouvait exister sans faire disparaître de la conscience la présence active de l'homme à l'origine de luimême » (Godelier, 1996, p. 240). Ceci signifie que le sacré au cœur du rituel sacrificiel est le moyen par lequel l'être social de l'homme se dérobe à luimême et engendre l'opacité dont il a besoin pour la production et la reproduction de la société.

\section{Organisation d'une production CAPRINE hÉRITÉE dU PASSÉ}

Si le bouc est objet de culte et de sacrifice et constitue en cela un fait religieux, il constitue également un fait économique. De la rencontre entre les fonctions religieuse et économique de l'animal, s'est progressivement instauré un type d'élevage dit d'arrière-cour. Ces pratiques d'élevage se sont sédimentées au cours de l'histoire jusqu'à devenir l'élément d'une tradition propre au territoire réunionnais. Celles-ci connaissent néanmoins des reformulations dans le cadre de la modernisation du secteur agricole avec le développement d'un élevage professionnel issu de l'adaptation de l'élevage traditionnel d'arrière-cour.

\section{Tradition d'élevage d'arrière-cour à la Réunion}

Durant la période coloniale, les propriétaires des domaines sucriers ont joué le rôle d'approvisionnement en boucs. Les temples se situaient alors sur les propriétés. Avec l'augmentation du niveau de vie pour les travailleurs des grands domaines, la possibilité d'entretenir des animaux s'est accrue, et ce tant pour les croyants et pratiquants que pour d'autres catégories aux origines sociales et culturelles diffé- rentes. L'élevage de boucs cérémoniels a alors pris place dans le cadre de la structure familiale, dans l'arrière-cour où l'on retrouve de petits élevages, comme l'exprime cet éleveur de caprins destinant sa production au rituel et se remémorant sa jeunesse : "Des particuliers, des petits éleveurs, il y en avait plein $[\ldots]$ Toutes les personnes avaient deux ou trois cabris. C'était d'abord pour leur consommation, mais après le surplus ils le vendaient. » La commercialisation s'est forgée par le bouche-à-oreille.

C'est ainsi que progressivement l'activité d'élevage à destination religieuse, est devenue une réalité qui va de soi pour tous les éleveurs aujourd'hui, ayant engendré chez eux au cours du temps une attention particulière à l'esthétique de l'animal qui recouvre deux dimensions principales. La première s'attarde à la prestance et à l'apparence de l'animal : « L'aspect, ça joue un rôle, c'est pour la religion. Quand le cabri, il présente bien, on se dit qu'on va le réserver pour les sacrifices. À sa présence aussi. La présence, c'est quand le cabri se présente bien debout ». La seconde concerne la couleur de la robe de façon à obtenir de préférence des animaux de couleur unie, blanche, noire, ou rouge, typique du bouc sacrificiel (figure 4). Cela se concrétise pour ceux qui le peuvent, ayant un cheptel suffisant, par un travail sur la dominance des caractères génétiques de la robe de l'animal : «Je fais des sélections pour obtenir un beau cabri rouge ou noir ». Cela se traduit aussi par une alimentation riche et accentuée dans les derniers mois précédant la vente de l'animal. L'emploi actuel de l'expression "bouc cérémonie » par les éleveurs eux-mêmes, fonctionne telle une solution permanente au problème que pose l'obligation religieuse de l'approvisionnement en boucs comme en témoigne l'extrait d'un entretien avec cet autre agriculteur «Si à La Réunion nous faisons ça [élever des boucs en vue de son utilisation religieuse], c'est parce qu'à La Réunion il y a des hindous. Et les hindous ont besoin de cabris depuis la nuit des temps ». Les éleveurs d'arrière-cour sont, ceux qui élèvent un nombre restreint d'animaux, achètent de jeunes boucs qu'ils font grossir pendant un à trois ans, avec une attention particulière portée à l'esthétique de l'animal, le but étant, soit de les vendre à destination du rituel sacrificiel hindou, soit de les élever dans le but de les offrir en sacrifice lorsqu'on est soi-même pratiquant. Ils sont réputés pour la qualité de l'animal élevé en vue du rituel « Vous allez 
voir à La Réunion dans les grands élevages, ce n'est pas là qu'il y a de jolis cabris. C'est là où il y en a 4 , $10 »$. L'animal est en effet considéré avoir atteint l'âge adulte vers trois ans. Or les éleveurs qui ont un cheptel plus important ont tendance à le vendre dans le cours de sa deuxième année en raison des coûts occasionnés par son entretien.

\section{Développement d'un élevage professionnel}

C'est au début des années 1980 qu'une autre catégorie d'éleveurs a commencé à se développer, pour pratiquer l'élevage de caprins dans le cadre d'une agriculture diversifiée ou source de revenus complémentaires. Cet élevage s'est instauré dans le contexte d'une évolution matérielle des conditions de production agricole qui le différencie des éleveurs d'arrière-cour. En effet, leur cheptel, plus conséquent, prend place à l'intérieur d'un ou de plusieurs bâtiments spécifiques. Le développement de cette situation s'accompagne par ailleurs chez les éleveurs d'une appropriation des représentations de l'activité d'élevage attribuant un caractère de pollution potentielle pour le voisinage caractéristique d'une vision urbaine : «Auparavant La Réunion était, on peut dire, à la campagne. Mais aujourd'hui c'est en train de se développer, c'est-à-dire que la construction ça pousse comme le champignon et donc on ne peut plus élever le cabri dans la cour à côté de son voisin, devant ou derrière. » Leur exploitation constitue généralement un agrandissement de ce qui était auparavant l'élevage d'arrière-cour des parents. Dans cette situation, leur production reste principalement destinée au rituel sacrificiel et à la commercialisation des animaux qui s'effectue toujours sur le mode du bouche-à-oreille. Depuis quelques années cependant, ces agriculteurs se sont regroupés en coopérative de production et d'approvisionnement. En 1997, des éleveurs installés depuis moins de cinq ans ont créé la coopérative des producteurs de caprins de la Réunion (CPCR). En 2000, le cheptel caprin a été dénombré à 21594 têtes dans 1787 élevages dits professionnels (Source : RGA, 2000). À cette date, on estimait également à 16000 têtes la production hors exploitations agricoles (source: Tableau économique de La Réunion, INSEE). La figure 1 présente la répartition géographique des cheptels de caprins selon les données par sous-communes du recensement de l'agriculture (Source: RGA, 2000).

Une étude réalisée en 2001, propose une typologie qui permet de distinguer 7 groupes d'éleveurs (Spaletta, 2 003) :

1 - les adhérents de la coopérative CPCR installés récemment ( 5 ans maximum); ces élevages sont en phase de développement; ils se situent dans les plaines à une altitude comprise entre 900 et $1600 \mathrm{~m}$ (ce groupe représente $26 \%$ des élevages enquêtés);

2 - des producteurs de canne à sucre dont l'élevage de caprins est une activité de diversification; le revenu caprin représente 10 à $50 \%$ des revenus du ménage (18\% des enquêtés);

3 - les gros élevages (30 à 150 mères) assurant plus de $50 \%$ du revenu des ménages (14\% des enquêtés);

4 - les élevages essentiellement naisseurs (14\% des enquêtés);

5 - les élevages de type basse-cour, de petite taille (1 à 10 mères) tournés vers l'autoconsommation; les frais d'élevage y sont réduits au maximum avec peu ou pas de concentré, un peu de foin et peu de traitements vétérinaires ( $12 \%$ des enquêtés);

6 - des personnes au RMI ou des salariés élevant des caprins et généralement d'autres animaux pour avoir un revenu supplémentaire (12\% des enquêtés);

7 - les naisseurs et engraisseurs de boucs : ce sont des éleveurs hindous ayant une activité d'achat et d'engraissement de boucs (4\% des enquêtés).

Sur les 1334 élevages professionnels recensés en 2007 (Source : enquête DAAF), on comptait 62 adhérents de la CPCR et deux éleveurs bovins allaitant adhérents de la Coopérative des producteurs de viandes (SICA REVIA) représentant seulement $5 \%$ des éleveurs adhérents à une coopérative (Boué et al., 2008).

Ces quelques données montrent que la production caprine reste caractérisée par l'élevage dit d'arrière-cour, avec de petits effectifs, en complément d'autres activités. Le secteur organisé passant par l'action de la CPCR demeure limité, mais enregistre une constante progression avec des retombées sur la commercialisation des animaux sur pied : 29,89 tonnes d'animaux sur pied en 2007 contre $21,25 \mathrm{t}$ en 2006 et $14 \mathrm{t}$ en 2005, soit un doublement en 2 ans (Boué et al., 2008). Le développement d'une agriculture organisée se substituant progressivement aux élevages d'arrière-cour se poursuit. 


\section{RÉGULATION GLOBALE ET ORDRE LOCAL : DES LOGIQUES EN CONFLITS}

Aujourd'hui, les pratiques d'élevage et d'abattage rituel sont mises en cause par l'application des règlements de sécurité sanitaire. Leur devenir se joue dans le projet de développement territorial de la filière caprine qui associe la Fédération des associations et groupements religieux hindous et culturels tamouls de la Réunion et les services de l'État que sont la DSV et la Préfecture, en charge de faire appliquer ces règlements. Dans ce projet, les deux types d'acteurs sont à la recherche d'un compromis. Pour maintenir le principe religieux fondamental du rituel sacrificiel de boucs, l'acteur associatif local revendique l'obtention d'une dérogation (arrêté préfectoral) permettant de maintenir les pratiques d'abattage rituel dans les temples publics et privés. L'administration sanitaire impose, d'une part, l'identification des animaux et, d'autre part, le référencement de la totalité des temples dans lesquels peuvent avoir lieu les sacrifices d'animaux. En coopérant avec la Fédération, l'attitude envisagée par l'administration est moins celle d'atteindre les éleveurs "disséminés » et méconnus par l'intermédiaire de la communauté religieuse, que de s'assurer, via la négociation, de l'identification des animaux destinés au rituel, gage de contrôle sanitaire. Dans ces conditions, si les animaux destinés au sacrifice sont systématiquement identifiés, les pratiques informelles d'élevage auront tendance à diminuer d'elles mêmes, car elles ne correspondront plus à la demande religieuse.

\section{Des règles sanitaires et des pratiques religieuses qui s'opposent}

L'arrêté du ministère de l'Agriculture et de la Pêche, daté du 19 décembre 2005, relatif à l'identification des animaux des espèces ovines et caprines, stipule que "les animaux doivent être identifiés par le détenteur-naisseur dans un délai de 6 mois à partir de leur naissance, et en tout état de cause avant leur départ de l'exploitation de naissance ». Ceci implique notamment l'obligation pour l'éleveur de déclarer tout animal possédé sous peine d'être soumis à la sanction d'une amende ou de se voir détruire quantité ou partie de son cheptel. Cet arrêté sous-entend également que l'animal soit « bouclé », c'est-à-dire percé et étiqueté d'un numéro de cheptel unique et ce sur les deux oreilles. Sachant que l'intégrité physique de l'animal doit être respectée, cette obligation vient à l'encontre des exigences religieuses se référant à l'esthétique de l'animal en vue du sacrifice.

Fondés avant tout sur des représentations à la fois scientifiques et techniques, les règlements sanitaires entrent en conflit avec la logique sacrificielle qui sous-tend le rituel. Le sang de l'animal en constitue un élément en étant perçu par les uns, les croyants, comme un des termes de la communication avec le monde du divin et par les autres, les services publics, comme potentialisateur de risque (Beck, 2001). Un des principaux enjeux concerne également la gestion des «sous-produits » générés lors des abattages d'animaux. Le sang et les abats sont notamment considérés comme des produits biologiques vecteurs potentiels de maladies et sources de pollution environnementale par les services vétérinaires. Dans le cadre de l'abattoir, le devenir de ces sous-produits est réglementairement prévu; ils sont généralement détruits par incinération. Or dans le cadre des abattages rituels, les abats peuvent être consommés par les participants, mais peuvent également être jetés dans des lieux considérés comme inappropriés par ces mêmes services vétérinaires : poubelles publiques, ravines ou rivières par exemple. Le corps des animaux sacrifiés est également transporté jusqu'au lieu où il va être préparé pour être cuisiné. Les participants ou bien le voisinage lorsque le lieu de préparation ne se trouve pas dans l'enceinte du temple peuvent donc être exposés.

\section{Vers la recherche d'un compromis}

L'ouverture en 2004, à Saint-Pierre, d'un abattoir (figure 1) pour les abattages rituels de bœufs pratiqués par les zarabs ${ }^{5}$ à l'occasion de la fête de l'Aid Al-Kabîr ${ }^{6}$ ainsi que l'actualisation, le $1^{\text {er }}$ janvier 2005, des règlements concernant l'identification des espèces ovines et caprines dans le cadre de l'union européenne, a fait prendre conscience à la Fédération des associations et groupements religieux hindous et culturels tamouls de La Réunion qu'il

5. Ce terme désigne en créole les descendants d'indiens musulmans.

6. La grande fête du sacrifice pour les Réunionnais de confession musulmane. 
fallait anticiper leur application en allant au devant des services de l'État; ceci afin d'obtenir une dérogation concernant les abattages rituels, en proposant en contrepartie de participer à la mise en place de la traçabilité à travers l'identification des animaux.

En effet, pour réduire les risques sanitaires liés aux pratiques religieuses, le secrétaire général de la Préfecture est en capacité d'interdire, par arrêté préfectoral, l'organisation de cérémonies comprenant des sacrifices d'animaux. Il lui est également possible de mobiliser les services vétérinaires, par définition sous la responsabilité de la Préfecture, pour des opérations de contrôles sur les lieux mêmes des cérémonies. Or, si on interdit les sacrifices d'animaux, c'est tout le système religieux qui est menacé ainsi que la possibilité de développer l'élevage caprin sur le territoire réunionnais (Ghasarian, 2010).

C'est pourquoi la Préfecture envisage d'ores et déjà d'habiliter certains temples, sur la base de critères artistiques, architecturaux et d'ouverture au public, dans le cadre desquels pourront avoir lieu les sacrifices d'animaux. Pour ce qui relève des cérémonies familiales, elles pourront avoir lieu à la seule condition d'une stricte consommation familiale conformément aux règlements sanitaires de l'autoconsommation des animaux. Mais, l'habilitation accordée à certains temples à l'exclusion des autres lieux de culte, dans le but de confiner le risque sanitaire à ces seuls endroits, remet en cause les formes de célébration généralisée sur l'île. D’autre part, les temples privés, ou Chapèl, accueillent les sèrvis familiaux à l'occasion desquels sont présents les membres de la famille restreinte certes, mais également des membres de la famille éloignée ainsi que des connaissances (figure 5). Ce type de célébration apparaît également menacé dans sa forme actuelle, et avec lui, les principes mêmes de solidarité et d'identité (Noujarede, 2007).

\section{Dans une société en reformulation permanente}

Cette situation de confrontation d'une norme sociétale et d'une norme religieuse hindoue mettant en scène le sacrifice animal est à rapprocher des études menées par une ethnologue française, AnneMarie Brisebarre, à propos des musulmans. Ses travaux portent sur le sacrifice du mouton pratiqué lors de l'Aid Al-Kabîr par les musulmans confrontés

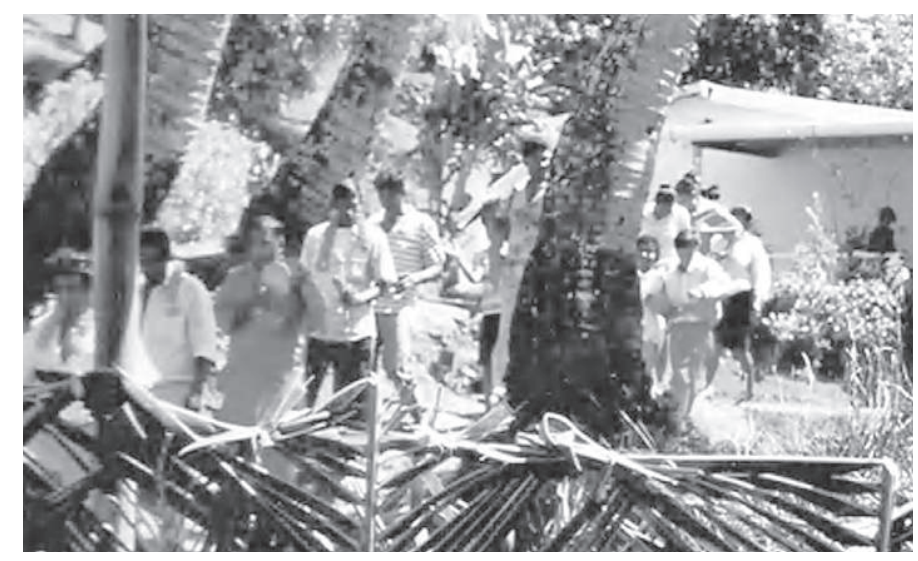

Figure 5 : Procession lors de la cérémonie domestique du sacrifice (sèrvis) Hindu procession at the family ceremony of sacrifice (sèrvis in Creole)

à l'évolution de leur propre société entrée dans la modernité (cas du Sénégal où le terme qui désigne cette fête en wolof est la Tabaski) (Julliard, 2010). Ils portent également sur le sacrifice pratiqué par les immigrés musulmans vivant en France et résidant en milieu urbain (Brisebarre, 1989). Ces recherches témoignent de deux passages majeurs : celui d'un animal « pur » de la tradition religieuse qui devient l'animal «propre » de la modernité et celui d'un culte majoritairement domestique qui devient de plus en plus un culte public sous le coup de la légalisation par le sacrifice de l'animal à l'abattoir. À la Réunion, l'évolution actuelle témoigne également de ces deux mouvements par l'urbanisation croissante d'une société historiquement déterminée par l'économie des plantations sucrières. En effet, depuis les années 1980, une classe moyenne réunionnaise urbaine liée à la tertiarisation de la société émerge, se substituant aux anciennes catégories engendrées par la Plantation (Ottino, 1999). L'urbanisation rend difficile aujourd'hui le sacrifice sur le lieu de résidence et les lois sanitaires le contrecarrent. Cependant, si en France métropolitaine notamment, la fête de l'Aid Al-Kabîr peut être perçue par une portion de la population française, comme une volonté de ne pas s'intégrer à la société, à La Réunion, le rituel sacrificiel du bouc est le fait d'une composante de la société et en cela est légitimée par la société elle-même. La recherche d'un compromis dans ce contexte traduit davantage la dimension plurielle que la société a acquise au cours du temps. La volonté politique actuelle d'administrer le secteur de 
la production caprine locale, tout en bouleversant les pratiques d'une production destinée principalement au rituel sacrificiel, ajoute à l'enjeu religieux celui qui combine le sanitaire et l'économie formelle par le développement d'une filière organisée. Face à l'enjeu de régulation globale, ce qui se passe à la Réunion autour de cette question témoigne de sa capacité à s'adapter et à se renouveler tout en maintenant les traits essentiels des cultures qui la composent.

\section{Conclusion : Acculturation ET STRATÉGIE IDENTITAIRE AU SERVICE DE LA PERPÉTUATION DU RITE}

Le sacrifice du bouc issu du culte populaire hindou en Inde s'est maintenu au cours de l'histoire à La Réunion. Au cœur d'une conception antagoniste de l'univers, entre des forces positives et des forces négatives sources de désordre, se sont forgés les principes de protection, de chance et de réussite, lesquels légitiment les manifestations concrètes de ce culte. De la rencontre entre la fonction religieuse et la fonction économique de l'animal, s'est instauré un type d'élevage dit d'arrière-cour. Plus récemment, s'est développé un autre type d'élevage constitué d'éleveurs organisés en coopérative dans la logique de modernisation de l'agriculture à La Réunion.

Aujourd'hui, les pratiques d'élevage et d'abattage rituel sont mises en cause dans le cadre de l'application des règles de sécurité sanitaire concernant les productions animales. Leur devenir se joue notamment dans le projet de développement d'une filière caprine organisée qui regroupe la Fédération des associations et groupements religieux hindous et culturels tamouls de la Réunion et les services de l'État, que sont la direction des Services Vétérinaires et la Préfecture, en charge de faire appliquer les règlements.

Pour l'acteur associatif hindou, ces règlements relèvent d'une forme d'ingérence dans ce qui relève des affaires sacrées. Pour autant, en même temps qu'il souhaite maintenir les éléments des principes culturels fondamentaux, tels que le rituel sacrificiel, il en ignore d'autres, notamment la perte de confiance dans l'activité d'élevage d'arrière-cour en faveur des éleveurs organisés qui acceptent de boucler les animaux pour garantir la traçabilité.
Pour les services de l'État, il s'agit d'une nécessité dont dépend le bien être collectif. Dès lors, entre enjeu de régulation globale et ordre local, ce face à face invite les croyants à adapter leurs pratiques religieuses et les éleveurs à modifier leurs pratiques agricoles au regard des règles sanitaires de santé publique.

Dans ce contexte de tension, potentiellement conflictuel, lié à l'application des règlements en matière d'élevage et d'abattage des animaux, la Fédération des associations et groupements religieux hindous et culturels tamouls de La Réunion se pose comme représentant religieux dans la négociation avec les services publics. Le fait de parler de traçabilité, d'identification des animaux, de risque sanitaire montre sa capacité à se penser par rapport aux catégories légitimées par la société, et témoigne des réajustements qui s'opèrent entre les deux modèles culturels. Il est également possible de constater que ces notions ont été également reprises et adoptées par les prêtres et par les éleveurs : «l'identification, les boucles, ce n'est pas un souci [...] c'est mieux pour pouvoir voir la traçabilité de l'animal si jamais il y a un problème de contamination ».

L'adoption de ces notions repositionne les principes fondamentaux de la culture dont les sacrifices d'animaux font partie. Ainsi, la Fédération souhaite organiser, anticiper les problèmes, assurer la protection sanitaire des participants, de manière à perpétuer le rituel qui a pour fonction d'assurer la bénédiction et la prospérité de l'ensemble de la communauté. Organiser la production agricole de caprins à destination du religieux tout en préservant le rituel implique la mise en place de nouvelles formes d'élevage garantissant l'établissement durable d'un réseau de connaissances, afin que les fidèles puissent se procurer suffisamment d'animaux.

Ainsi, sous l'effet conjugué de l'évolution des modes de vie et du développement d'une agriculture modernisée, ainsi qu'au nom du principe de précaution qui sous-tend la gestion du risque sanitaire, les services publics incitent au développement de l'élevage organisé au détriment de l'élevage d'arrièrecour. Mais au stade actuel, si l'organisation de la production caprine fondée sur les éleveurs organisés et les temples publics est en passe de devenir cet ensemble au service de la perpétuation du rite, et si elle s'accompagne d'une diminution de l'élevage caprin d'arrière-cour, on ne peut présager pour 
autant de la disparition de ce dernier. Car, par son caractère de petit élevage celui-ci demeure le plus à même de travailler les aspects d'une esthétique positive de l'animal adaptés aux exigences religieuses.

Dans la négociation qui s'est engagée, c'est l'habilitation des temples publics en vue du rituel qui a été retenue, au détriment des autres lieux. Cette solution envisagée, les cérémonies aux lieux de culte domestiques privés (chapèls) apparaissent menacées et avec elles leur inscription sociale et spatiale par le rôle d'activation des réseaux familiaux et de proximité qu'elles remplissent.

Ainsi, la confrontation des modèles culturels et la recherche de compromis à l'échelle territoriale sont des conditions d'évolution et de transformation sociales dans nos sociétés contemporaines entre régulation globale et ordre local. Au-delà du projet de filière de production caprine où s'est instaurée la confrontation entre l'acteur religieux et les services publics, il convient désormais d'observer les effets de ce qui a été discuté et proposé sur les pratiques réelles tant sur le plan religieux que sur le plan des activités d'élevage. Dans quelle mesure le maintien des identités locales par les processus sociaux engendrés dans la confrontation va-t-il aboutir à des formes renouvelées de pratiques religieuses et de pratiques économiques?

\section{Remerciements}

Merci à Philippe Lecomte responsable de l'UR Système d'élevage et produits animaux $d u$ Cirad qui a accueilli Edouard Noujarede en stage de Master dans le cadre du Projet ANR-06-Padd-0017 Spaldd (2006-2010). Merci à tous ceux qui ont participé à l'étude. Merci à Christian Ghasarian ainsi qu'à Maurice Godelier pour leur soutien à la réflexion anthropologique. Merci à Tom Wassenaar pour l'extraction des données cartographiques. Merci enfin aux deux relecteurs pour leurs commentaires et propositions de références qui nous ont permis d'enrichir la rédaction de cet article.

\section{Bibliographie}

BЕск U., 2001. Société du risque : sur la voie d'une autre modernité, Paris, Aubier, 521 p.

Boué P., Sigwald J.-P., Jousset M., 2008. Mission d'expertise sur l'amélioration génétique des caprins dans les départements d'Outre-Mer et à Mayotte, ODEADOM et Capgènes, 50 p.

Brisebarre A.-M., 1989. La célébration de l'Ayd el-Kébir en France : les enjeux du Sacrifice, Archives des sciences sociales des religions, vol. 1, $\mathrm{n}^{\circ}$ 68, p. 9-25.

Ghasarian C., 1991. Honneur, Chance et Destin. La culture indienne à La Réunion, Paris, l'Harmattan, $256 \mathrm{p}$.

GHasarian C., 2002. La Réunion : acculturation, créolisation et réinventions culturelles : Vers une anthropologie du postcolonialisme?, Ethnologie française, Vol. 32, n 4, p. 663-676.

Ghasarian C., 2010. Coup pa nout tradition! Des sacrifices hindous face aux régulations européennes, in LE GALL D. et Roinsard N. (dir.), Chroniques d'une autre France. Genres de vie et intimités créoles, vol 4, Paris, L'Harmattan, p. 61-83.

Godelier M., 1996. L'énigme du don. Paris, Fayard, 315 p.

Julliard A. (dir.), 2010. La Tabaski au Sénégal. Une fête musulmane en milieu urbain, Archives de sciences sociales des religions, document 152-22, mis en ligne le 17 mai 2011, [http://assr.revues.org/22658].

Live Y.-S., 1999. Sociologie de La Réunion : Mutations, paradoxes, représentations, migrations (quelques pistes de recherche), in ChÉRubini B. (dir.), La recherche anthropologique à La Réunion. Vingt années de travaux et de coopération régionale, Paris, L'Harmattan, p. 185-203.

Mauss M., 1985. Esquisse d'une théorie générale de la magie, in Mauss M., Sociologie et anthropologie, Paris, PUF (4 édition), p. 2-141.

Noujarède E., 2007. Le sacrifice de boucs à La Réunion. Actes rituels, organisation de marché et compromis culturels. Mémoire de master de sociologie, UCO-IPSA, Angers, 76 p.

Otтino P., 1999. Quelques réflexions sur les milieux créoles réunionnais, in ChÉrubini B. (dir.), La recherche anthropologique à La Réunion. Vingt années de travaux et de coopération régionale, Paris, L'Harmattan, p. 65-95.

Piet L., 2005. La construction socio-juridique de la traçabilité des viandes bovines : entre politique sanitaire et organisation de marché (1960-2002), Cahiers d'économie et de sociologie rurale, $\mathrm{n}^{\circ} 74$, p. 109-138.

Robineau C., 1994. Anthropologie et économie de marché, Cahiers des sciences humaines, $\mathrm{n}^{\circ} 30, \mathrm{p}$. 23-33.

Spaletta V., 2003. Le marché du cabri à La Réunion, INRA, CIRAD, ESITPA, La Réunion, 34 p.

VAXelaire D., 2009. L'histoire de La Réunion. De 1848 à nos jours, vol 2, La Réunion, Editions Orphie (2e édition), $703 \mathrm{p}$.

Wyatт V. H., 2009. Brucellosis and Maltese goats in the Mediterranean, Journal of Maltese history, $\mathrm{n}^{\circ}$ 2, p. 4-18. 